\title{
Reactive Arthritis: Recent Advances and Clinical Manifestations
}

Key words: reactive arthritis, spondyloarthropathy, HLAB27, streptococcus

\section{Seronegative spondyloarthropathy and reactive arthri- tis}

Joint pain (arthralgia/ arthritis) is associated with various physical conditions and various rheumatic or non-rheumatic diseases. Rheumatoid factor (RF) and antinuclear antibody (ANA) are good markers of screening for rheumatic diseases. However, there are many rheumatic diseases in which either RF or ANA is not demonstrated in patients' sera. Therefore, it is important to precisely inquire about the patients' past history and present illness precisely and perform a careful physical examination in order to achieve the proper diagnosis.

"Reactive arthritis" is classified as one type of seronegative spondyloarthropathy (SNSA), which consists of ankylosing spondylitis (AS), reactive arthritis (ReA; Reiter's syndrome), psoriatic arthritis and arthritis associated with chronic inflammatory bowel diseases. Typical clinical presentations are RF negative in their sera, axial joint arthritis (sacroiliitis, spondylitis), enthesitis and peripheral oligoarthritis. In many patients, the subtalar, metatarsophalangeal and toe interphalangeal joints tend to be affected. The number of affected peripheral joints is typically less than 5 (oligoarthritis) and are usually asymmetrically involved in the lower limbs. The muscloskeletal symptoms are generally acute in onset associated with constititutional symptoms such as fatigue, malaise, fever and weight loss. The arthritis is usually quite painful. Dactylitis, or "sausage digit", a diffuse swelling of a solitary finger or toe, is a distinctive feature of both ReA and psoriatic arthritis (1-3) (Figs. 1, 2).

\section{Extra-articular manifestations}

Tendinitis and fasciitis are characteristic lesions, producing pain at multiple insersion sites, especially the Achilles tendon (Fig. 3), the plantar fascia, and sites along the axial skeleton. Spinal and low-back pain are common features which are caused by insertional inflammation, muscle spasm, acute sacroiliitis or arthritis in intervertebral articulations.

Mucocutaneous manifestations are highly associated in ReA. Keratoderma blennorrhagium is a papulosquamous skin rash that appears most commonly on the sole or palms (Fig. 4). Circinate balanitis is the characteristic lesion involv-

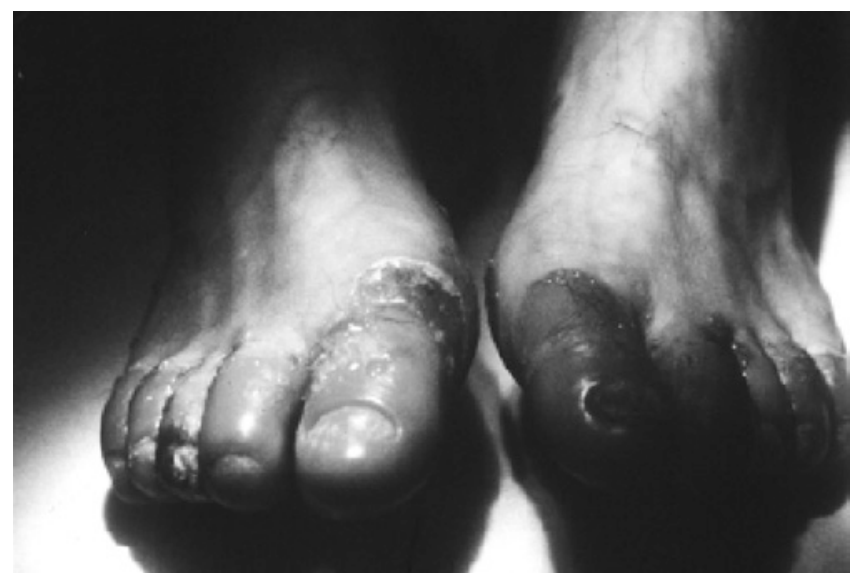

A

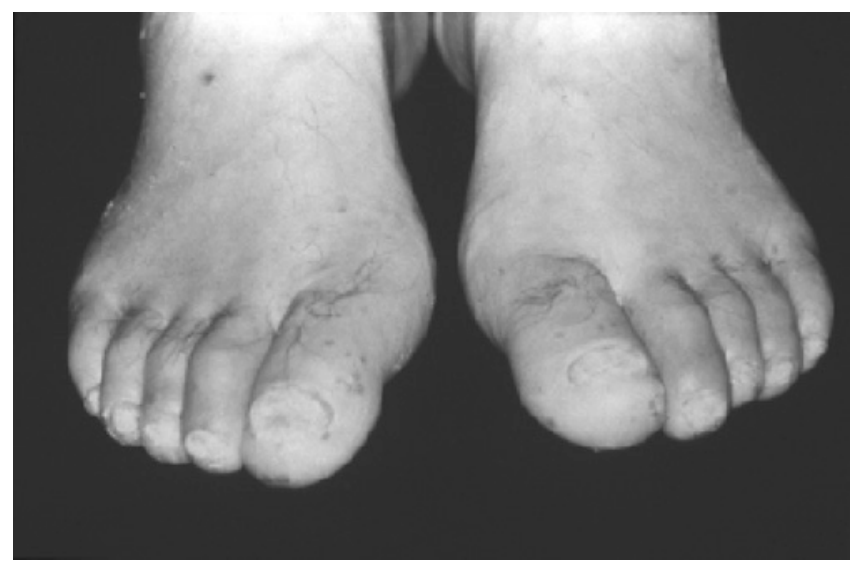

B

Figure 1. Dactylitis in Chlamydia-induced reactive arthritis. A: Before treatment, B: After treatment.

ing the glans or shaft of the penis. Oral ulcers, which are shallow and usually painless, may appear on the tongue or hard palate. Acute anterior uveitis occurs in nearly $20 \%$ of patients.

\section{Involvement of microorganism infection}

Among SNSA, it is an important clinical manifestation that either urogenital or bowel infection is characteristically involved in the development of ReA. Therefore, we should ask patients whether they had an episode of urethritis or 


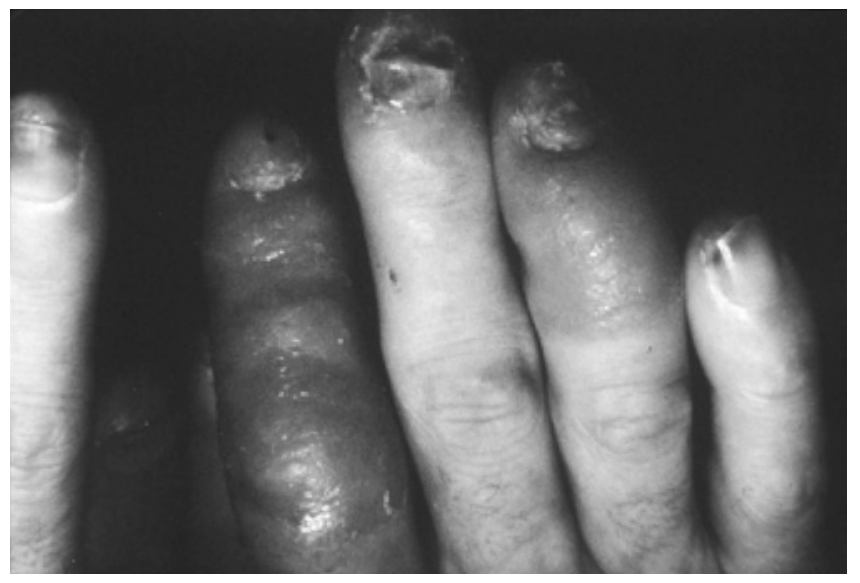

A

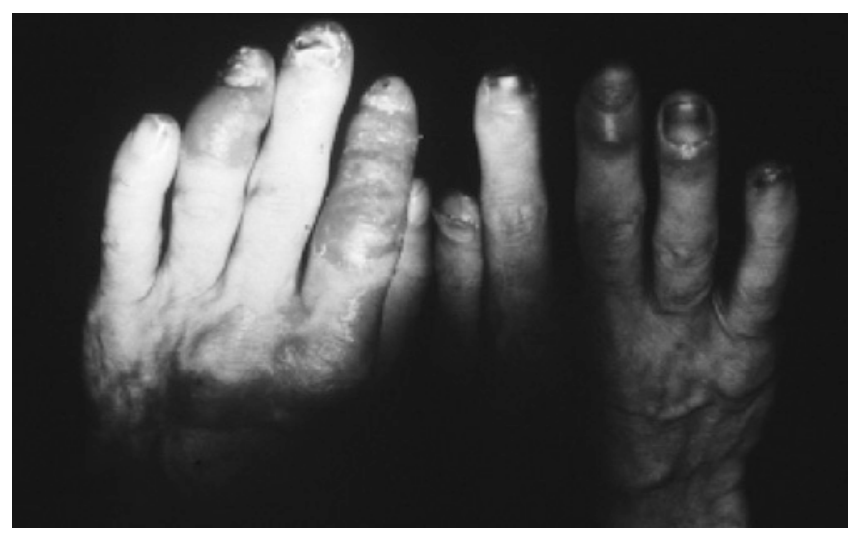

B

Figure 2. Sausage digits in Chlamydia-induced reactive arthritis.

diarrhea within 4 weeks prior to the appearance of arthritis. Chlamydia trachomatis in urogenital infection, Salmonella enteritidis, Yersinia enterocolitica, Shigella flexneri and Campylobactor jejuni in bowel infection are microorganisms which are known to induce $\operatorname{ReA}(1-3)$. Episodes of Chlamydia-induced urethritis are sometimes subtle and often asymptomatic as a case report presented in this issue (4). Therefore, we inquire about any untypical sexual intercourse or ask whether the patient found stain in their underwear.

See also p 509.

\section{Relation to HLA-B27}

Typing for HLA-B27 is positive in approximately $70 \%$ of Caucasian patients with ReA. Around 7-14\% of Caucasian population are positive for HLA-B27, however, less than $1 \%$ are positive in the Japanese population. Therefore, the incidence or prevalence of AS or ReA is quite low in Japan as compared to that in the USA or Europe. HLA-B27 is more

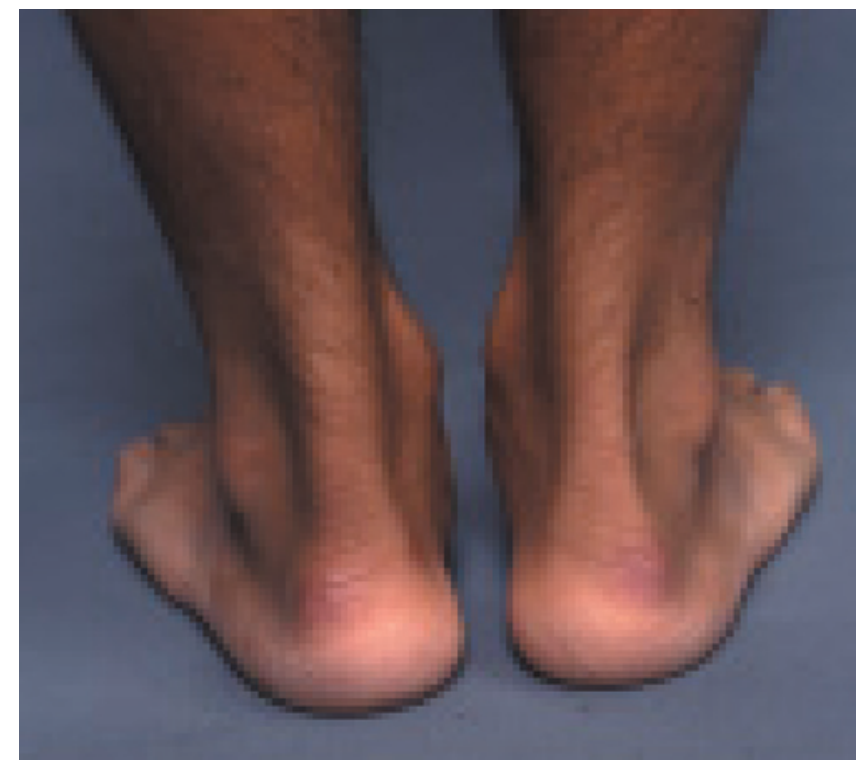

Figure 3. Enthesitis: inflammation of the left Achilles tendon insertion area. Yersinia enterocolitica-induced reactive arthritis.

likely to be found in patients with complicating sacroiliitis, and spondylitis. Therefore, Japanese patients with psoriatic arthritis and arthritis associated with chronic inflammatory bowel diseases, who have only peripheral joint arteritis, are seldom found to have HLA-B27. In addition to HLA-B27, HLA-B7 and B60 are HLA-B27-related antigens. It has been reported that the majority of HLA-B27 negative Japanese AS patients have HLA-B39 which is molecular homologous to HLA-B27 (5). Reactive arthritis after streptococcal infection of the pharynx or tonsils is reported as post-streptococcal reactive arthritis (PSRA) or tonsillitis-associated reactive arthritis (Fig. 5). This form of reactive arthritis is a different clinical entity from so-called HLA-B27-related "reactive arthritis". The authors found that HLA-B39 was determined in the majority of patients with tonsillitis-associated reactive arthritis (6).

HLA molecules in general, and class I molecules in particular, evolved in response to specific environmental stresses, and polymorphisms of HLA class I molecules deal with infectious stresses, such as viruses and bacteria. HLAB27-positive individuals are associated with less efficient intracellular elimination of Chlamydia and certain enteric bacteria than HLA-B27-negative individuals. Therefore, the HLA-B27 molecules may contribute to disease susceptibility due to its failure to eliminate intracellular organisms or by presentation of arthrogenic peptides.

\section{Proposed classification criteria and laboratory tests determining Chlamydia}

Proposed classification criteria and laboratory tests which are recommended for determining Chlamydia are listed in Tables 1, $2(3,7)$. 

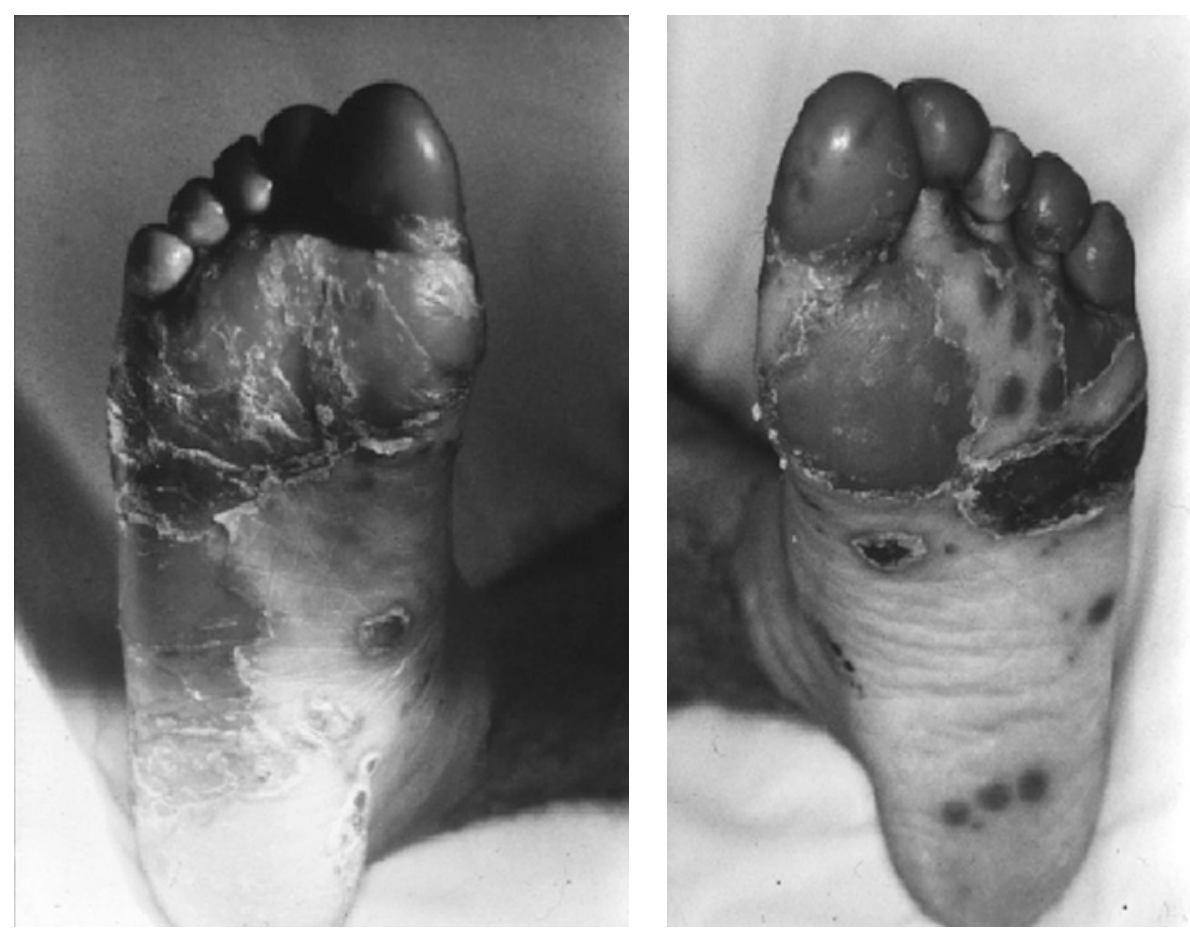

Figure 4. Keratoderma blennorrhagium in Chlamydia-induced reactive arthritis.
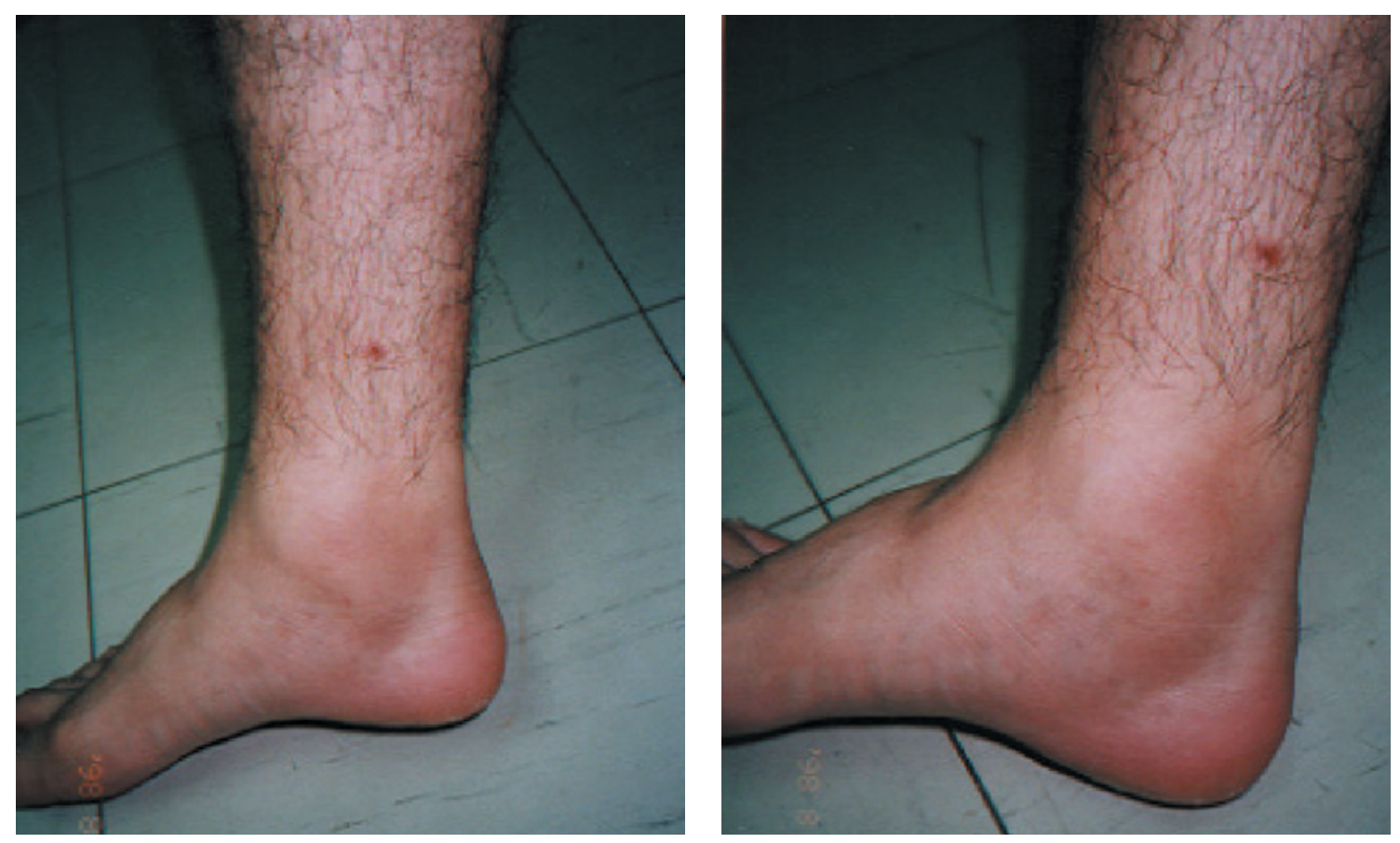

Figure 5. Arthritis at ankle joints in patients with post-streptococcal reactive arthritis. 
Major Criteria

1. Arthritis (2 of 3$)$

Asymmetric

Mono or oligoarthritis

Lower limbs

2. Preceding symptomatic infection (1 of 2)

Enteritis (diarrhea for 1 day, 3 days to 6 weeks before infection)

Urethritis (dysuria/discharge for 1 day, 3 days to 6 weeks before infection)

Minor Criteria (1 of 2)

1. Evidence of triggering infection

Positive urine LCR or urethral/cervical swab for Chlamydia trachomatis

Positive stool culture for ReA related enterobacteria

2. Evidence of persistent synovial infection (A positive immunobistology is generally conceivable, but is not routinely used, as too laborious)

Positive PCR for Chlamydia

Additional Classification

Urogenic or enteric ReA

Acute $(\leq 6 \mathrm{mo})$ and chronic $(>6 \mathrm{mo}) \mathrm{ReA}$

Exclusion Criteria

Other defined rheumatic diseases should be excluded by clinical history and examinatin and by performing the following tests as a minimum:

Where synovial fiuid is available, microscopy, culture, and crystal analysis

Serology for rheumatoid factor, antinuclear antibodies and, where appropriate, anti-Borrelia burgdorferii and antistreptococcal antibodies

Radiology for chondrocalcinosis, joint space narrowing

Definition of Reactive Arthritis

Definitie ReA: First and second major criteria and a relevant minor criterion

Probable ReA: First and second major criteria, but no relevant minor criteria

or

First major criterion and one or more minor criteria

The authors think that these are so-called "diagnostic criteria" but not "classification criteria" since the criteria have "exclusion criteria" and subclassification of "definite ReA" and "Probable ReA". The style is similar to the ACR 1953 diagnostic criteria for RA.

Table 2. Laboratory Tests a Rheumatologist should Perform in Determining the Diagnosis of ReA

1. In case of a preceding symptomatic infection in the urogenital tract

Obligatory

Urethral/cervical smear (for Chlamydia) or

Morning urine (for Chlamydia)

Facultative

Chlamydia serology?

HLA-B27?

Synovial fluid PCR (Chlamydia)?

No Yersinia or Salmonella serology is recommended.

2. In case of a preceding symptomatic infection in the enteral tract

Obligatory

Stool culture (enterobacteria)

Yersinia, Salmonella serology

Facultative*

HLA-B27?

No urethral smear, no morning urine, no Chlamydia serology, and no SF PCR are recommended.

3. In case of no preceding symptomatic infection

Morning urine (for Chlamydia)

Urethral smear (for Chlamydia)

Yersinia, Salmonella serology

Facultative*

HLA-B27?

Chlamydia serology?

SF PCR?

No stool cultures are recommended.

*Recommended by only one-third of the experts. SF: synovial fluid. 


\section{Treatment}

For Chlamydia-induced ReA, prompt, appropriate antibiotic treatment of acute urethritis is necessary and will prevent subsequent reactive arthritis. Non-steroidal anti-inflammatory drugs benefit most patients to some degree. Sulfasalazine is beneficial to patients with persistant ReA. Immunosuppressive agents such as azathioprine or methotrexate are also effective. Intra-lesional glucocorticoid injection is beneficial for tendonitis and for other enthesitic lesions.

Shigeto KoBAYASHI and Issei KIDA

Department of Internal Medicine, Juntendo Koshigaya Hospital, 560 Fukuroyama, Koshigaya, Saitama 343-0032

\section{References}

1) Taurog JD, Lipsky PE. Ankylosing spondylitis, reactive arthritis, and undifferentiated spondyloarthropathy. 4th Ed. Harrison's Principles of Internal Medicine. Fauci AS, Braundwald E, Isselbacher KJ, et al Eds. McGraw-Hill, Tokyo, 1998: 1904-1909.

2) Spieper J, Braun J. Reactive arthritis. Curr Opin Rheumatol 11: 238243, 1999.

3) Spieper J, Braun J, Kingsley GH. Report on the Fourth International Workshop on Reactive Arthritis. Arthritis Rheum 43: 720-734, 2000.

4) Ishii $W$, Matsuda $M$, Okamoto $N$, et al. Reactive arthritis due to asymptomatic infection of Chlamydia trachomatis. Intern Med 44: 509-510, 2005.

5) Yamaguchi A, Tsuchiya N, Mitsui $\mathrm{H}$, et al. Association of HLA-B39 with HLA-B27-negative ankylosing spondylitis and pauciarticular juvenile rheumatoid arthritis in Japanese patients. Evidence for a role of the peptide-anchoring B pocket. Arthritis Rheum 38: 1672-1677, 1995.

6) Kobayashi S, Tanaka M, Tamura N, Yamamoto S, Akimoto $T$, Hashimoto H. Tonsillitis induced reactive arthritis. Arthritis Rheum $\mathbf{3 5}$ suppl 9: s244, 1992.

7) Spieger J, Rudwaleit M, Braun J, van der Heijde D. Diagnosing reactive arthritis. Role of clinical setting in the value of serologic and microbiologic assays. Arthritis Rheum 46: 319-327, 2002. 